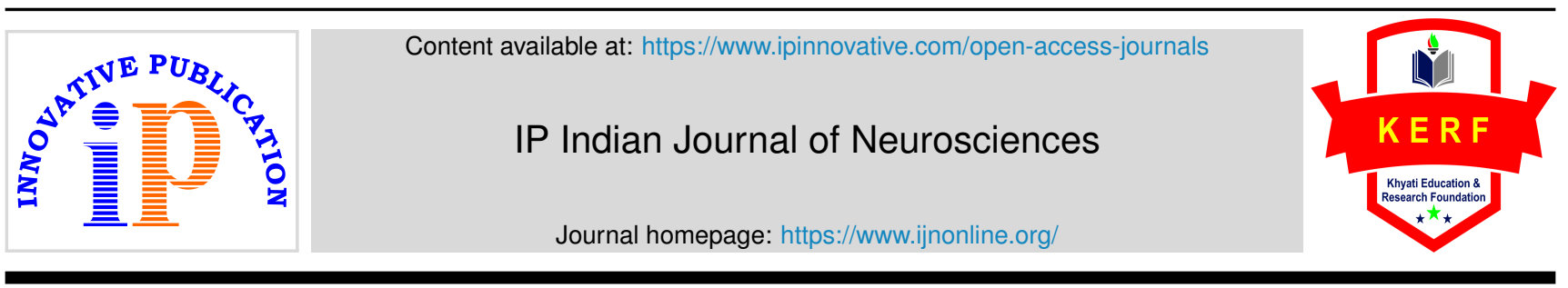

Original Research Article

\title{
A study of short term and long term risk of ischemic stroke and other vascular events following early treatment of tia and minor stroke in a tertiary centre in North India
}

\author{
Shashidhar Manchala ${ }^{1} *$, Vinod Kumar Mehta ${ }^{1}$, Sitaram Barath $^{\mathbf{2}}$ \\ ${ }^{1}$ Dept. of Neurology, Geetanjali Medical College and Hospital, Udaipur, Uttar Pradesh, India \\ ${ }^{2}$ Dept. of Radiology, Geetanjali Medical College and Hospital, Udaipur, Rajasthan, India
}

\section{A R T I C L E I N F O}

\section{Article history:}

Received 12-05-2021

Accepted 24-05-2021

Available online 14-07-2021

\section{Keywords:}

TIA

Ischemic Stroke

Minor Stroke

\begin{abstract}
A B S T R A C T
Introduction: Transient ischemic attack and minor ischemic stroke are associated with early recurrence and deterioration respectively. There is a high risk of stroke after TIA ranging between $10-20 \%$ in the ensuing 90 days in the various studies. There are various clinical and imaging factors which predict the early risk of stroke after a TIA.

Aim of the study: To evaluate the short term and long term risk of ischemic stroke following early treatment of TIA and minor stroke.

Materials and Methods: TIA is defined as sudden focal neurologic deficit of presumed vascular origin lasting less than 24 hours. Minor ischemic stroke is defined as National institute of Health Stroke scale score of $\leq 5$. TIA mimics were excluded from the study. The patients were followed up till 1 year. In patients with minor ischemic stroke, a 2-point worsening in the NIHSS was considered as an event. Modified Rankin score at 3 months was assessed.

Results: Total 320 patients were analysed with a mean age of $60.6 \pm 13.6$ years. Out of the them $20.7 \%$ were females, $78 \%$ of patients had minor stroke. There is significant association of lacunar stroke in minor stroke patients $(\mathrm{p}<0.01)$, whereas most TIA patients, etiology remained undetermined. In multiple regression analysis, both diabetes $[\mathrm{p}<0.05]$ and significant vessel stenosis $(>50 \%)[\mathrm{p}<0.05]$ were found to be an independent predictor of recurrent stroke in TIA and minor stroke patients.

Conclusion: Urgent treatment after TIA and minor ischemic stroke is associated with reduced risk of ischemic stroke recurrence. Both diabetes mellitus and significant vessel stenosis are independent predictor of stroke recurrence.
\end{abstract}

(C) This is an open access article distributed under the terms of the Creative Commons Attribution License (https://creativecommons.org/licenses/by/4.0/) which permits unrestricted use, distribution, and reproduction in any medium, provided the original author and source are credited.

\section{Introduction}

Transient ischemic attack and minor ischemic stroke are associated with early recurrence and deterioration respectively. There is a high risk of stroke after TIA ranging between $10-20 \%$ in the ensuing 90 days in the various studies. But recent studies have found urgent interventions to be associated with substantial reductions in stroke risk within the first 90 days following TIA $(0.6 \%$ to $3.1 \%)$. There are various clinical and imaging factors which predict

\footnotetext{
* Corresponding author.

E-mail address: drshashidhar484@gmail.com (S. Manchala).
}

the early risk of stroke after a TIA. Identifying the stroke subtype also help in estimating the risk of stroke recurrence and also the risk of deterioration after a minor stroke. Large artery atherosclerosis has been found to be associated with a high risk of stroke after a TIA. A metanalysis of four population based studies of stroke showed that the risk of recurrent stroke was $4 \%$ (95\% CI: $0.2-7.8)$ at 7 days and $12.6 \%$ (95\% CI: 5.9-19.3) at 30 days in patients with large artery atherosclerotic aetiology compared to 0 and $2 \%(95 \%$ CI 0 -4.2) respectively in patients with lacunar stroke. In addition to the short-term risk of stroke, these patients are at increased long-term risk of ischemic vascular events. We 
know that urgent evaluation and treatment of these patients is associated with significant reduction of the short-term risk of stroke, but there was not much literature available about the effect of early treatment and long-term outcome. The purpose of the study is to evaluate short term and long-term outcome and their predictors in patients with TIA or minor ischemic stroke who receive urgent intervention.

\section{Aim of the study}

1. To evaluate the short term and long term risk of ischemic stroke following early treatment of TIA and minor stroke

2. To evaluate the occurrence of non stroke vascular events following early treatment of TIA and minor stroke.

\section{Methods and Materials}

Patients with TIA and minor ischemic stroke admitted in stroke unit of Geetanjali Medical College, Udaipur within 48 hours of onset and patients seen as outpatient within 48 hours of symptom onset from November 2016 to October 2020. TIA mimics were excluded from the study. The patients were followed up till 1 year.

\subsection{Study design}

The proposed study is a retrospective cohort study of patients with TIA and minor ischemic stroke admitted in stroke unit of Geetanjali Medical College, Udaipur. within 48 hours of onset and patients seen as outpatient within 48 hours of symptom onset from November 2016 to October 2020. TIA is defined as sudden focal neurologic deficit of presumed vascular origin lasting less than 24 hours. Minor ischemic stroke is defined as National institute of Health Stroke scale score of $\leq 5$. TIA mimics were excluded from the study. The patients were followed up till 1 year.

The primary outcome is stroke or TIA at 3 months and at one year. In patients with minor ischemic stroke, a 2point worsening in the NIHSS will be considered as an event. The Modified Rankin score at 3 months is assessed. The secondary outcome will be any non stroke vascular events or vascular deaths at 3 months and at one year. Non-stroke vascular event will include any Myocardial infarction, unstable angina and peripheral vascular disease leading to amputation of that limb occurring during follow up.

Clinical and socio demographical data, National Institutes of Health Stroke Scale scores and Modified Rankin Scale scores, as well as data on vascular risk factors were collected from patients. The vascular risk factors include

1. Diabetes

2. Hypertension
3. Age

4. Smoking

5. Renal dysfunction

6. Dyslipidemia

7. Peripheral vascular disease

8. Coronary artery disease

\section{Statistical analysis}

All statistical analysis was performed using SPSS software. Continuous variables are reported as mean \pm SD. Categorical variables were reported as proportions. Baseline characteristics were compared between the 2 groups. Differences in continuous variables were assessed by 1-way analysis of variance and differences between proportions were assessed by the $\mathrm{X}^{2}$ test. Univariate and multivariate analysis was done to identify predictors of new vascular events. Multiple logistic regression model was used to identify independent predictors of new vascular events.

\section{Results}

Total 320 patients were analysed with a mean age of $60.6 \pm$ 13.6 years. Out of the them $20.7 \%$ were females.

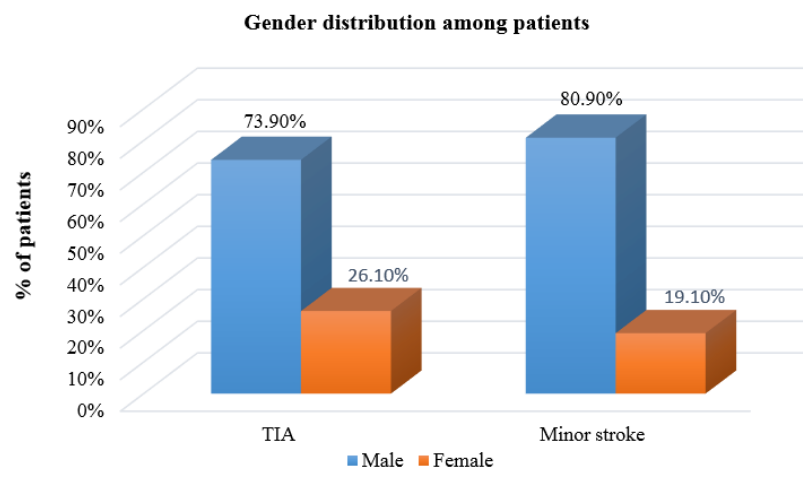

Fig. 1: Sex distribution of the study group

Table 1 Out of 320 patients, $78 \%$ of patients had minor stroke.

Table 2 Among the study group, 198 patients (61.9\%) had Hypertension, 138 patients $(43.1 \%)$ had Diabetes, 137 patients $(42.8 \%)$ had dyslipidaemia and $34(10.6 \%)$ had atrial fibrillation.

\subsection{Comparison of baseline characteristic between TIA and Minor stroke patients}

There is no significant difference between demographic characteristic, risk factor association and clinical characteristic between TIA and minor stroke patients. Limb weakness $(63.4 \%)$ was the most common in both groups of patients followed by speech impairment (dysarthria in $46.3 \%$ and aphasia in $12.8 \%$ patients). There is significant association of DWI positivity and minor stroke patients compared to TIA patients $(\mathrm{p}<0.01)$. 
Table 1: Risk factors associated with TIA and Minor stroke patients

\begin{tabular}{lccc}
\hline Risk Factor & TIA & Minor stroke & Total \\
Hypertension & $43(62.3 \%)$ & $155(61.8 \%)$ & $198(61.9 \%)$ \\
DM & $23(33.3 \%)$ & $115(45.8 \%)$ & $138(43.1 \%)$ \\
Smoking & $19(27.5 \%)$ & $66(26.3 \%)$ & $85(26.6 \%)$ \\
CAD & $9(13 \%)$ & $41(16.3 \%)$ & $50(15.6 \%)$ \\
Atrial Fibrillation & $4(5.8 \%)$ & $30(12 \%)$ & $34(10.6 \%)$ \\
Valvular heart disease & $5(7.2 \%)$ & $30(12 \%)$ & $35(10.9 \%)$ \\
Dyslipidaemia & $28(40.6 \%)$ & $109(43.4 \%)$ & $137(42.8 \%)$ \\
Peripheral Vascular Disease & 0 & $10(4.1 \%)$ & $4(3.1 \%)$ \\
(PVD) & & &
\end{tabular}

Table 2: Comparison between baseline characteristic between TIA and Minor stroke patients

\begin{tabular}{|c|c|c|c|c|}
\hline & TIA & Minor stroke & Total & P value \\
\hline No. of patients & $69(21 \%)$ & $251(79 \%)$ & 320 & \\
\hline Age & $62.6 \pm 13.8$ & $59.4 \pm 13.5$ & $60.6 \pm 13.6$ & 0.13 \\
\hline $\operatorname{Sex}(M: F)$ & $51: 18$ & 203:48 & $254: 66$ & 0.2 \\
\hline Hypertension & $43(62.3 \%)$ & $155(61.8 \%)$ & $198(61.9 \%)$ & 0.9 \\
\hline DM & $23(33.3 \%)$ & $115(45.8 \%)$ & $138(43 \%)$ & 0.05 \\
\hline Smoking & $19(27.5 \%)$ & $66(26.3 \%)$ & $85(26.6 \%)$ & 0.83 \\
\hline CAD & $9(13 \%)$ & $41(16.3 \%)$ & $50(15.6 \%)$ & 0.5 \\
\hline Atrial Fibrillation & $4(5.8 \%)$ & $30(12 \%)$ & $34(10.6 \%)$ & 0.14 \\
\hline Valvular heart disease & $5(7.2 \%)$ & $30(12 \%)$ & $35(10.9 \%)$ & 0.26 \\
\hline Dyslipidaemia & $28(40.6 \%)$ & $109(43.4 \%)$ & $137(42.8 \%)$ & 0.6 \\
\hline $\begin{array}{l}\text { Peripheral Vascular } \\
\text { Disease (PVD) }\end{array}$ & 0 & $10(4.1 \%)$ & $4(3.1 \%)$ & 0.09 \\
\hline Limb weakness & $45(65.2 \%)$ & $158(62.98 \%)$ & $203(63.4 \%)$ & 0.7 \\
\hline Dysarthria & $31(44.9 \%)$ & $117(46.6 \%)$ & $148(46.3 \%)$ & 0.8 \\
\hline Aphasia & $7(10.1 \%)$ & $34(13.5 \%)$ & $41(12.8 \%)$ & 0.4 \\
\hline MRI DWI positive & $29(48.3 \%)$ & $169(92.3 \%)$ & $198(61.8 \%)$ & $<0.01$ \\
\hline $\begin{array}{l}\text { MRI DWI multiple } \\
\text { positive }\end{array}$ & $11(18.3 \%)$ & $53(29.0 \%)$ & $64(26.3 \%)$ & 0.1 \\
\hline $\begin{array}{l}\text { Significant vessel } \\
\text { stenosis }(>50 \%)\end{array}$ & $12(15.4 \%)$ & $66(26.3 \%)$ & $78(24.4 \%)$ & 0.12 \\
\hline
\end{tabular}

Table 3: Distribution of etiologic subtypes of TIA and Minor stroke patients

\begin{tabular}{lcccc}
\hline Etiologic subtypes & TIA & Minor stroke & Total & P value \\
LAA & $9(13 \%)$ & $38(15.1 \%)$ & $47(15 \%)$ & 0.6 \\
Lacunar & $6(8.7 \%)$ & $81(32.6 \%)$ & $87(27.2 \%)$ & $<0.01$ \\
Cardioembolism & $7(10.1 \%)$ & $51(20.3 \%)$ & $58(18.1 \%)$ & 0.05 \\
Undetermined & $47(68.1 \%)$ & $72(28.6 \%)$ & $119(37.2 \%)$ & $<0.01$ \\
Other determined & 0 & $9(3.6 \%)$ & $9(2.8 \%)$ & 0.11 \\
\hline
\end{tabular}

\subsection{Etiologic subtypes of TIA and Minor stroke patients}

Table 2 According to TOAST classification, in 47(15\%) patients, the cause of stroke was large artery atherosclerosis, in $58(18.1 \%)$ cardioembolism, in $87(27.2 \%)$ lacunar, in $9(2.8 \%)$ other determined etiology and in 119 (37.2\%) undetermined cause. There is significant association of lacunar stroke in minor stroke patients $(\mathrm{p}<0.01)$, whereas most TIA patients, etiology remained undetermined. In the large artery atherosclerosis group, 32(68\%) of patients had intracranial atherosclerosis. Carotid endarterectomy was performed in 8 patients.

\subsection{New events in TIA and stroke patients at 3 months and 1 year}

Table 3 New cerebrovascular event occurred in 23 $(7.7 \%)$ and 25 patients $(8.5 \%)$ at 3 months and 1 year respectively. 14 out of 23 events $(60 \%)$ occurred within 3 days of occurrence of stroke. There is no significant difference between TIA and minor stroke patients regarding association of new events. In case of cardiovascular event, 7 and 13 patients $(2.2 \%$ and $4.4 \%)$ respectively, had new events at 3 months and 1 year. There were 5 deaths in 3 months of stroke, out of which 2 were vascular death and one patient died due to intracranial hemorrhage who was 
Table 4: New cerebrovascular and cardiovascular events in TIA and Minor stroke patients

\begin{tabular}{lcccc}
\hline & TIA & Minor stroke & Total & P value \\
No. of patients & $69(21 \%)$ & $251(79 \%)$ & 320 & 0.11 \\
Cerebrovascular event at 3 & $2(2.9 \%)$ & $21(8.4 \%)$ & $23(7.2 \%)$ & 0.26 \\
months & & & & $25(8.5 \%)$ \\
Cerebrovascular event at 1 year & $3(4.9 \%)$ & $22(9.4 \%)$ & $7(2.2 \%)$ & 0.65 \\
Cardiovascular event at 3 months & $2(2.9 \%)$ & $5(2 \%)$ & $13(4.4 \%)$ & 0.35 \\
Cardiovascular event at 1 year & $4(6.6 \%)$ & $9(3.8 \%)$ & \\
\hline
\end{tabular}

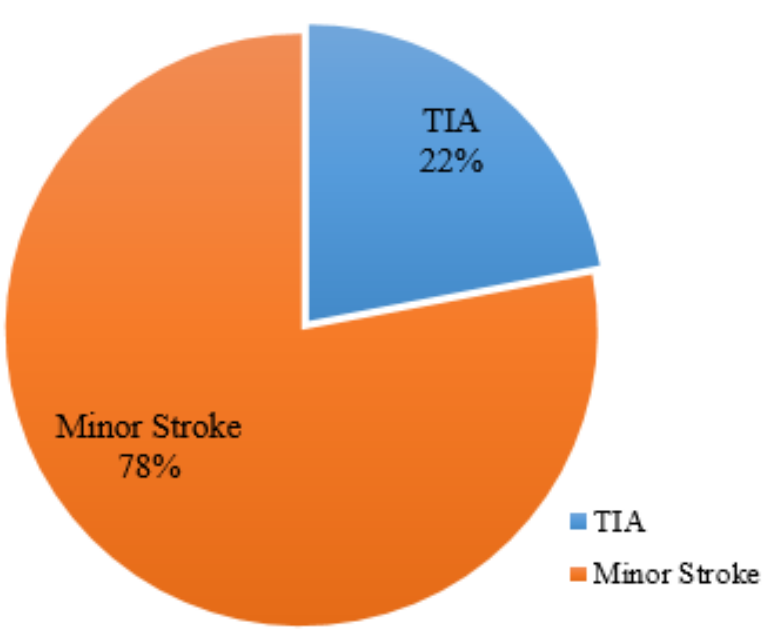

Fig. 2: Proportion of patients with TIA and minor stroke

on anticoagulant. 2 other patient died due to non-vascular causes.

\subsection{Predictors of recurrence of stroke at 3 months}

Table 4 In univariate analysis both diabetes mellitus and significant vessel stenosis $(>50 \%)$ were significantly associated with recurrence of stroke. All patients who had recurrent event had ABCD2 score $\geq 4$.

Table 5 In multiple regression analysis, both diabetes [OR- 2.6 CI (1.1-6.6), p<0.05] and significant vessel stenosis $(>50 \%)$ [OR- 3.1 CI $(1.3-7.6), \mathrm{p}<0.05]$ were found to be an independent predictor of recurrent stroke in TIA and minor stroke patients.

\subsection{Outcome of stroke at 3 months}

14 out of 23 patients $(60 \%)$ who had recurrence of stroke had significant disability $(\mathrm{mRS} \geq 2)$, whereas 53 patients $(17.8 \%)$ who do not have recurrence of stroke had significant disability at 3 months. During these periods only 6 patients received IV thrombolysis, out of which one patient had significant disability (mRS 3), whereas other patient had good outcome (mRS 0 or 1 ). No patient had symptomatic intracranial hemorrhage.
Table 5: Predictors of recurrence of stroke

\begin{tabular}{lcc}
\hline Factors & $\begin{array}{c}\text { Stroke } \\
\text { recurrence }\end{array}$ & P value \\
Age (>60 years) & $9(5.3 \%)$ & 0.16 \\
Sex & $20: 3$ & 0.35 \\
Hypertension & $17(8.6 \%)$ & 0.22 \\
DM & $15(10.9 \%)$ & 0.01 \\
Smoking & $5(5.9 \%)$ & 0.59 \\
CAD & $4(8.1 \%)$ & 0.8 \\
Valvular heart disease & $4(11.4 \%)$ & 0.31 \\
Atrial Fibrillation & $3(8.8 \%)$ & 0.7 \\
Peripheral Vascular Disease & $2(20 \%)$ & 0.11 \\
Dyslipidemia & $10(7.3 \%)$ & 0.96 \\
ABCD ( $\geq 4)$ & $23(100 \%)$ & 0.11 \\
Multiple DWI lesion & $4(6.3 \%)$ & 0.7 \\
Significant vessel stenosis & $11(14.1 \%)$ & 0.01 \\
(>50\%) & & \\
LAA & $6(12.8 \%)$ & 0.1 \\
Lacunar & $8(9.2 \%)$ & 0.4 \\
CE & $7(12.1 \%)$ & 0.11 \\
\hline
\end{tabular}

\section{Discussion}

Our study investigated the short term and long-term recurrence of ischemic stroke in TIA and minor stroke patients in a tertiary care centre in north India, where round the clock stroke service is available. The study population consisted of 320 patients out of which $21 \%$ of patient had TIA.

Patients were classified according to TOAST classification. In our study in TIA group, $13 \%, 8.7 \%$ and $10.1 \%$ of patients were having large vessel atherosclerosis, lacunar and cardioembolism as etiology whereas in $68 \%$ of patient's etiology remained undetermined. Levelle et $\mathrm{al}^{1}$ in their study showed comparable frequencies [26\%, $4.1 \%$ and $10.5 \%$ patients were having large artery atherosclerosis, lacunar and cardioembolism as etiology, whereas in $51 \%$ of patient's etiology remained undetermined]. Similarly, in the minor stroke group, $15.1 \%, 32.6 \%$ and $20.3 \%$ of patients were having large vessel atherosclerosis, lacunar and cardioembolism as etiology. In $28.6 \%$ of patients the etiology was undetermined. Previous study done by $\mathrm{Wu}$ et $\mathrm{al}^{2}$ reported $41.8 \%, 22.4 \%$ and $2.8 \%$ patients were having large artery atherosclerosis, lacunar and cardioembolism as etiology of stroke. In the study, $28.8 \%$ of patients the etiology remained undetermined. Overall in our study, 
$14.7 \%, 27.2 \%$ and $18.1 \%$ of patient had large vessel atherosclerosis, lacunar and cardioembolism as etiology of stroke. These differences can be explained by regional differences in risk factors and different study population.

Hypertension $(62.3 \%$ in TIA and $61.8 \%$ in minor stroke, overall $61.9 \%$ of patients), diabetes mellitus (33.3\% in TIA and $45.8 \%$ in minor stroke, overall $42.2 \%$ of patients) and dyslipidemia $(40.6 \%$ in TIA and $43.4 \%$ in minor stroke, overall $42.8 \%$ of patients) were the vascular risk factors most commonly associated with study population. Levelle et $\mathrm{al}^{1}$ in their study also reported similar association of risk factors with study population.

In our study limb weakness (63.4\%) and speech impairment $(55 \%)$ were the predominant clinical symptoms presented by the patient which were same as reported by Levelle et al ${ }^{1}$ in their study.

The study showed a recurrence of ischemic stroke in $2.9 \%$ and $8.4 \%$ of patients with TIA and minor stroke at 3 months. Overall, recurrence rate of cerebrovascular event at 3 months is $7.2 \%$ which was similar to the rate of stroke recurrence reported in a recent metanalysis $(6.7 \%$ $(\mathrm{CI}-5.2-8.7 \%)){ }^{3}$ The recurrence rate is higher for minor stroke patients compared to TIA patients. Most of the stroke recurrence $(60 \%)$ occurred within 3 days, suggesting the importance of urgent evaluation and treatment of these patients. In our study, stroke recurrence rate at one year is $8.5 \%$ which was lower compared to a metanalysis which reported risk of recurrent of stroke at $>90$ days to be $11.3 \%$ (CI 7.5-16.6\%). ${ }^{3}$ But a study by Amarenco et al, showed that the 3 months and 1 year stroke recurrence rate was 3.7 and $5.1 \%$, which was lower compared to our study. ${ }^{4}$ This may be related to geographic variation, resource availability and health seeking behaviour of peoples of developing countries.

In our study $2.2 \%$ and $4.4 \%$ of patients had new cardiovascular event at 3 months and 1-year suggestive high risk of association of cardiovascular event with TIA and minor stroke patients. Previous studies also found similar rate of occurrence of cardiovascular event in TIA and minor stroke patients. ${ }^{5-7}$

All our patients with TIA and minor stroke, who had stroke recurrence had ABCD2 score of 4 or more, suggesting a high sensitivity but poor specificity for prediction of new vascular event. Wardlaw et al in a recent metanalysis reported similar findings (sensitivity $86.7 \%$ and specificity $35.4 \%)^{8}$

In our study, increasing age was not associated with increased risk of stroke in TIA and minor stroke patients. This contrasts with earlier study by Jhonston et al ${ }^{9}$ where they reported age as a predictor of stroke recurrence. But Ois et al in their study had found age was not significantly associated with further stroke recurrence in both TIA and minor stroke patients. ${ }^{10}$
Among vascular risk factor, diabetes mellitus was found to be an independent predictor of stroke recurrence in our patient. Similar findings had been reported by Chen et al in their study as both diabetes and metabolic syndrome independently associated with increased stroke risk in TIA and minor stroke patient. ${ }^{11}$ However, in our study we have not evaluated the association of metabolic syndrome with stroke recurrence.

In our study, both intracranial and extracranial significant vessel stenosis $(>50 \%)$ found to be an independent predictor of stroke recurrence at 3 months, irrespective of the stroke subtype. Coutts et al in their study also reported similar findings as significant vessel stenosis as a strong predictor of stroke recurrence. ${ }^{12}$

Our study reported stroke recurrence rate as $12.8 \%$, $12.1 \%$ and $9.2 \%$ in large artery atherosclerosis, cardioembolism and lacunar stroke subtype respectively. We do not find any significant association between stroke recurrence and individual stroke subtype. This contrasts with findings reported by most of the previous studies where a strong association was found with large artery atherosclerosis and early recurrence of stroke. ${ }^{4,10}$ But Ay et al in their study did not find large artery atherosclerosis as independent predictor of recurrent stroke in TIA patients. ${ }^{13}$ Previous study done by Lovett et al reported as $19.2 \%, 11.9 \%$ and $3.4 \%$ recurrence rate of stroke in LAA, cardioembolism and lacunar etiology. ${ }^{14}$ Ohara et al have reported high risk of recurrent stroke (mostly lacunar) in TIA patients without LAA or AF which may be specific to Asian populations compared to western population. ${ }^{15}$ In our study also there is higher recurrence of stroke in lacunar group compared to western data. The low risk of recurrent stroke in large artery atherosclerosis in our population may be explained by geographic variation, early intervention in the form of early revascularization surgery and optimum medical management and lower prevalence of intracranial $(32 \%)$ than extracranial stenosis.

In our study, $60 \%$ of patient who had recurrence of stroke had significant disability ( $\mathrm{mRS} \geq 2$ ), whereas $17.8 \%$ who do not have recurrence of stroke had bad outcome at 3 months. This is in accordance with the study done by Coutts et al, suggesting that a substantial portion of patients remained disabled after TIA and minor stroke. ${ }^{12}$ During these periods only 6 patients received IV thrombolysis, out of which one patient had significant disability (mRS 3), whereas rest of the patient had good outcome (mRS 0 or 1 ). No patients had symptomatic intracranial hemorrhage.

\subsection{Strength of the study}

The strength of the study is early inclusion of the patient within $48 \mathrm{~h}$ of the index event. Acute stroke patients were selected because these are the patients who are likely evaluated in detail in the stoke care unit. This is one of the largest studies from north India which focuses on 
the outcome of early treatment of TIA and minor stroke. Patients were evaluated by stroke neurologist, mimics were excluded from study as far as possible.

\subsection{Limitation of the study}

It was a retrospective study so chances of bias are high. Moreover, the record for compliance of treatment were not available. Only those patients presented within 48 hours of index event were included in the study, hence patient present late were not studied.

\section{Conclusion}

Our study showed that urgent treatment after TIA and minor ischemic stroke is associated with reduced risk of ischemic stroke recurrence. They are also having increased risk of both short term and long term cardiovascular events subsequently. Both diabetes mellitus and significant vessel stenosis are independent predictor of stroke recurrence. We have also found that a substantial portion of patient remained disabled at 3 months.

The role of IV thrombolysis in decreasing disability needs to be validated with further study. Patients, who have significant vessel stenosis being at highest risk of disability in view of recurrent event, early intervention will be most beneficial in this group of patients.

\section{Source of Funding}

No financial support was received for the work within this manuscript.

\section{Conflicts of Interest}

There are no conflicts of interest.

\section{References}

1. e PCL, Meseguer E, Abboud H. A transient ischaemic attack clinic with round-the- clock access (SOS-TIA): feasibility and effects. Lancet Neurol. 2007;6(11):953-60.

2. Wu L, Wang A, Xianwei W, Zhao X, Wang C, Liu L, et al. on behalf of the China National Stroke Registry investigators. Factors for short-term outcomes in patients with a minor stroke: results from China National Stroke Registry. BMC Neurol. 2015;15:253.

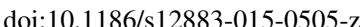

3. Wardlaw J, Brazzelli M, Miranda H. A systematic review and metaanalysis of stroke risk after transient ischaemic attack. In: NIHR J Libr. vol. 27; 2014. p. 17-32.

4. Amarenco P, Lavallee PC, Labreuche J. One-Year Risk of Stroke after Transient Ischemic Attack or Minor Stroke. $N$ Engl J Med .
2016;374(16):1533-42. do1:10.1016/1.jVS.2016.08.067,

5. Touzé E, Varenne O, Chatellier G, Peyrard S, Rothwell PM, Mas $\mathrm{JL}$, et al. Risk of Myocardial Infarction and Vascular Death After Transient Ischemic Attack and Ischemic Stroke. Stroke.

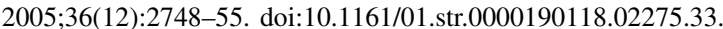

6. Gongora-Rivera F, Labreuche J, Jaramillo A, Steg PG, Hauw JJ, Amarenco P, et al. Autopsy Prevalence of Coronary Atherosclerosis in Patients With Fatal Stroke. Stroke. 2007;38(4):1203-10.

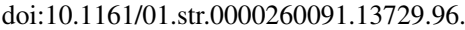

7. Rokey R, Rolak LA, Harati Y, Kutka N, Verani MS. Coronary artery disease in patients with cerebrovascular disease: A prospective study. Ann Neurol . 1984;16(1):50-3. 10i:10.1002/ana.410160س0.

8. Wardlaw JM, Brazzelli M, Chappell FM. ABCD2 score and secondary stroke prevention: meta-analysis and effect per 1,000 patients triaged. Neurology. 2015;85:373-80.

9. Johnston SC, Gress DR, Browner WS, Sidney S. Short-term prognosis after emergency department diagnosis of TIA. JAMA. 2000;284:29016.

10. Ois A, Gomis M, Rodríguez-Campello A, Cuadrado-Godia E, Jiménez-Conde $\mathrm{J}$, Pont-Sunyer $\mathrm{C}$, et al. Factors Associated With a High Risk of Recurrence in Patients With Transient Ischemic Attack or Minor Stroke. Stroke. 2008;39(6):1717-21.

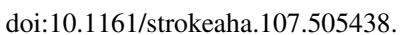

11. Chen W, Pan Y, Jing J, Zhao X, Liu L, Meng X, et al. Recurrent Stroke in Minor Ischemic Stroke or Transient Ischemic Attack with Metabolic Syndrome and/or Diabetes Mellitus. J Am Heart Assoc.

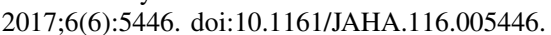

12. Coutts SB, Modi J, Patel SK, Aram H, Demchuk AM, Goyal M, et al. What causes disability after transient ischemic attack and minor stroke? Results from the CT and MRI in the Triage of TIA and minor Cerebrovascular Events to Identify High Risk Patients (CATCH) Study. Stroke. 2012;43:3018-22.

13. Ay H, Arsava EM, Johnston SC, Vangel M, Schwamm LH, Furie KL, et al. Clinical- and Imaging- based Prediction of stroke risk after transient ischemic attack. The CIP models. Stroke. 2009;40:181-6.

14. Lovett JK, Coull A, Rothwell PM. Early risk of recurrent stroke by aetiological subtype: Implications for stroke prevention. Neurology. 2004;62:569-74.

15. Ohara T, Uehara T, Toyoda K, Suzuki R, Sato S, Nagatsuka $\mathrm{K}$, et al. Early Stroke Risk after Transient Ischemic Attack in Patients without Large-Artery Disease or Atrial Fibrillation. J Stroke Cerebrovasc Dis. 2015;24(7):1656-61. doi:10.1016/1.jstrokecerebrovasdis.2015.03.039.

\section{Author biography}

Shashidhar Manchala, DM Resident

Vinod Kumar Mehta, Associate Professor

Sitaram Barath, Associate Professor

Cite this article: Manchala S, Mehta VK, Barath S. A study of short term and long term risk of ischemic stroke and other vascular events following early treatment of tia and minor stroke in a tertiary centre in North India. IP Indian J Neurosci 2021;7(2):138-143. 